\title{
Operation strategy for a low-cost easy-operation Cassino Hexapod
}

doi:10.1080/11762320802002573

\author{
Giuseppe Carbone, ${ }^{1}$ Aviral Shrot ${ }^{2}$ and Marco Ceccarelli ${ }^{1}$ \\ ${ }^{1}$ Laboratory of Robotics and Mechatronics, DiMSAT-University of Cassino, Via Di Biasio 43, 03043 Cassino (Fr), \\ Italy \\ ${ }^{2}$ Department of Mechanical Engineering, Indian Institute of Technology Madras, Chennai 600036, India
}

\begin{abstract}
This paper presents operation strategies for a hexapod walking machine that has been designed and built at the Laboratory of Robotics and Mechatronics (LARM) at the University of Cassino. Special care has been addressed in proposing and describing a suitable mechanical design and architecture that can be easily operated by a PLC with on-off logic. Experimental tests are reported in order to show feasibility and operational capability of the proposed design.
\end{abstract}

Key words: Robotics, walking machines, design, programming.

\section{INTRODUCTION}

Design of intelligent walking machines that can move in uneven terrains and areas which are inaccessible to humans has been a challenge in robotics for a very long time (Rosheim, 1994). Stable, efficient and fast robots (Salmi and Halme, 1996; Hirose et al., 1997) are needed to navigate uneven environments which can help humans in areas such as demining (Rachkov et al., 2005), pipe inspection (Kawaguchi et al., 1995; Horodinca et al., 2002), inspection and restoration of archaeological sites (Ceccarelli et al., 2002; Cigola et al., 2005) and interplanetary exploration (Wilcox et al., 1992).

Walking machines can be of different types. Some are based on crawlers or wheels. Others are equipped with biologically inspired legs (Molinaro, 2005). This type of walking machines can be slow and more difficult to design and operate with respect to the previous ones. Nevertheless, legged robots are more suitable for rough terrain, where obstacles of any size can appear (Carbone and Ceccarelli, $2004 a, 2004 b)$. In fact, the use of wheels or crawlers limits the size of the obstacle that can be climbed to half the diameter of the wheels (Chakraborty and Ghosal, 2004). On the contrary, legged machines can overcome obstacles that are comparable with the size of the machine leg (Carbone and Ceccarelli, 2005; Hirose and Yoneda Robotic Lab Webpage). There is also a third type of waking machines that is called hybrid since it has legs and wheels at the same time (Intelligent Machines and Special Robotics Institute Web-

Corresponding author:

Giuseppe Carbone

Email: carbone@unicas.it page). This type of walking machines (Berns) may range from wheeled devices to true walking machines with a set of wheels. In the first case, the suspensions are arms working like legs to overcome particularly difficult obstacles. In the second case wheels are used to enhance the speed when moving on flat terrain.

In this paper, the Cassino Hexapod walking robot design and operation are presented. The operation strategy and programming is discussed together with preliminary laboratory tests. This robot is controlled digitally by using a commercial PLC.

Main novelties of the work are the low-cost and easy-operation features for a complex hexapod walking machine. Such a complex system usually requires high-level knowledge of control hardware and software. Instead, a suitable mechanical design with suitable sensors and actuators gives the possibility to be more user-friendly and reduces the costs. In fact, even non-engineers users can easily learn how to operate the system while the control hardware and software have lower costs. These low-cost and easy-operation design features widen the number of potential users of the proposed hexapod walking machine. The paper attempts to describe the main issues in the mechanical design and hardware for achieving the above-mentioned goals. Then, the operation strategy for the proposed hexapod design is reported with the well-known Grafcet discrete control method in order to demonstrate the easy-operation features.

\section{ATTACHED PROBLEM}

A hexapod walking robot consists of six legs. Proper movement of the robot is possible by properly synchronising the 


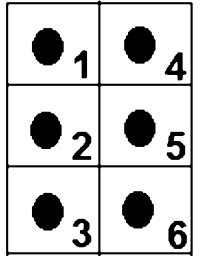

a)

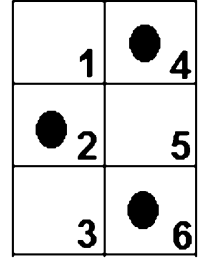

b)

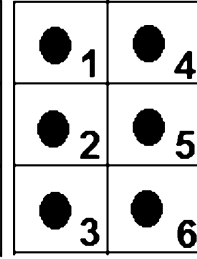

c)

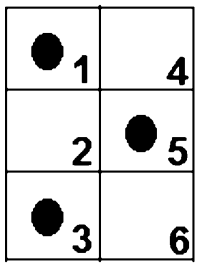

d)
Figure 1 Scheme of steps in hexapod gait of Cassino Hexapod: (a) all legs in contact with the ground; (b) legs 1-3-5 are lifted together and moved forward; (c) all legs come in contact with the ground momentarily; (d) legs 2-4-6 are lifted together and moved forward.

motion of all the legs. Thus, programming the movement of one leg is very important for the successful movement of the robot. Each leg joint of the Cassino Hexapod is actuated by a DC motor which is activated by PLC, based on the digital logic. Motion of a particular joint stops as soon as it reaches its extreme position, which is sensed by the limit switches.

Suitable walking gait has been implemented for the locomotion of Cassino Hexapod. For maintaining the stability of the robot, at least three legs must be in contact with the ground simultaneously (Zhao et al., 2000; Zielinska and Heng, 2002; Carbone and Ceccarelli, 2005). In the tripod gait, the front and the rear leg of one side and the middle leg of another side perform their swing movements at the same time. Thus the swings of right and left tripods have to be synchronised by adding a software-induced delay. This has to be done to implement a continuous walk. The scheme of hexapod walking gait of Cassino Hexapod has been shown in Figure 1. Thus, the attached problem has considered how to achieve a suitable easy programming for a hexapod gait through low-cost programmable power units and fairly simple programming of PLC using on-off logic.

\section{MECHANICAL DESIGN AND HARDWARE OF A PROTOTYPE OF CASSINO HEXAPOD}

A single-link module can be defined as a link module which contains in itself the needed actuators, transmissions and sensors which can be connected together to form a mechanical leg. The leg consists of several single-link modules. Each module consists of a separate motor, hence increasing the number of links which results in increasing the number of degrees of freedom (dofs) of the robot. For our purposes we have used three links to compose a single leg, as shown in Figure 2. Each leg has four dofs, the fourth being arranged for the wheeled foot.

The leg in Figure 2 consists of three modules; $1_{1}, l_{2}$ and $l_{3}$, each of which is driven by a motor, represented by $R_{1}, R_{2}$ and $R_{3}$ (as shown in Fig. 2a). Another motor $R_{4}$ is used as the power source for the wheeled foot, enabling the

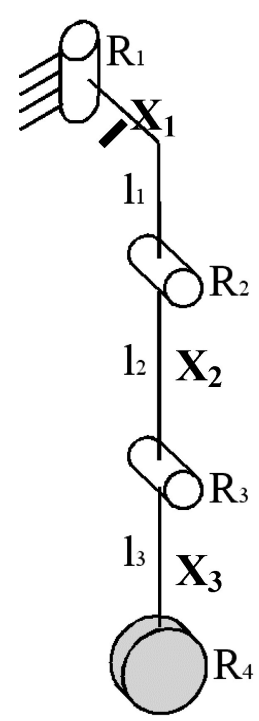

a)

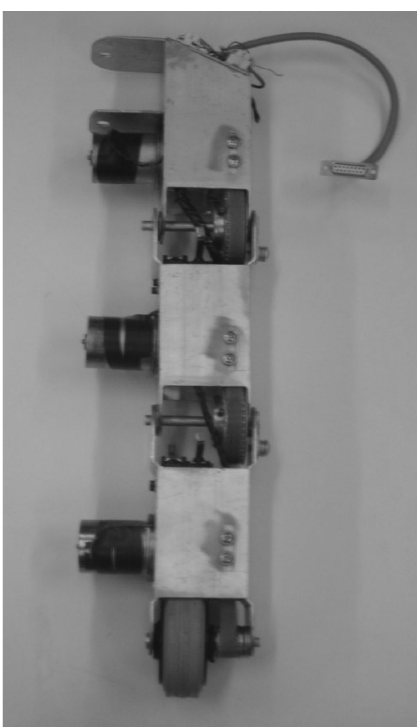

b)
Figure 2 The Cassino modular leg with three dofs: (a) a kinematic scheme; (b) a built prototype at LARM.

smooth movement of the hexapod in a flat environment. The motor $R_{1}$ can be modelled as the hip joint, for turning the leg inside and out. The motor $R_{2}$ is used to lift the leg up and down and finally the motor $R_{3}$ is equivalent to the knee joint.

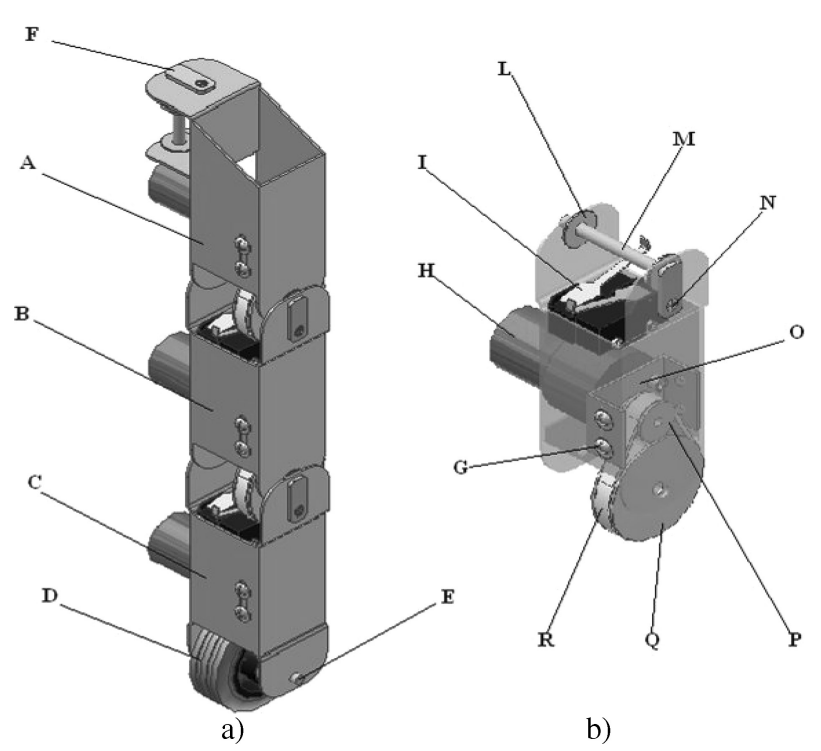

Figure 3 A 3D CAD model with main components: (a) one leg with three dofs; (b) a standard module. (A: modified module to be connected with base frame; B: standard module; C: modified module with wheel; D: wheel; F: pin for revolute joint with lock-up clutch; G: screws; H: motor with reduction gearbox; I: limit switches; L: washer; M: pin for revolute joint with lock-up clutch; N: screw for fixing lock-up clutch; O: fixing frame for motor; P: pulley on input shaft; Q: pulley on output shaft; R: belt.) 
Each motor is equipped with a corresponding limit switch $\mathrm{X}_{i}$, which signals to stop the motion of the motor when joint limit is reached.

The robotic leg has been made up of commercially available components, which have been assembled together to give the final form of the leg that is shown in Figure 2(b). The weight of each anthropomorphic leg is $2.5 \mathrm{~kg}$.

Figure 3 reports main components that have been selected and used for assembling a robotic leg having three dofs. The proposed design has been conceived for developing a walking leg by using mainly low-cost industrial components with the following basic requirements:

- to have a robust simple mechanical design;

- to have a modular design that can be used for robots with different number of legs;

- to be operated with an easy flexible programming;

- to have low-cost both in design and operation.

The above-mentioned requirements can be achieved in a very practical way by using low-cost components from the market into a suitable design for the whole system. In particular, the components that are indicated with D, E, G,

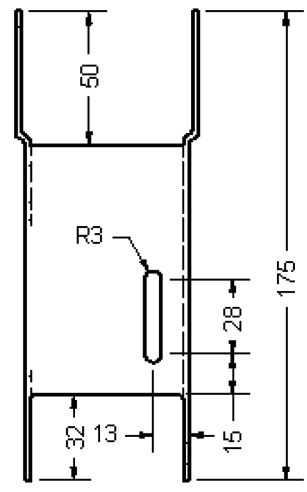

a)

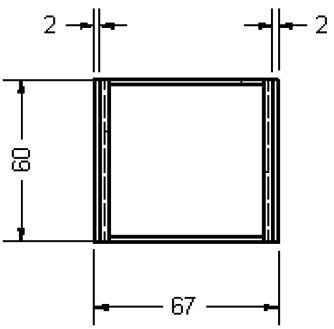

c)

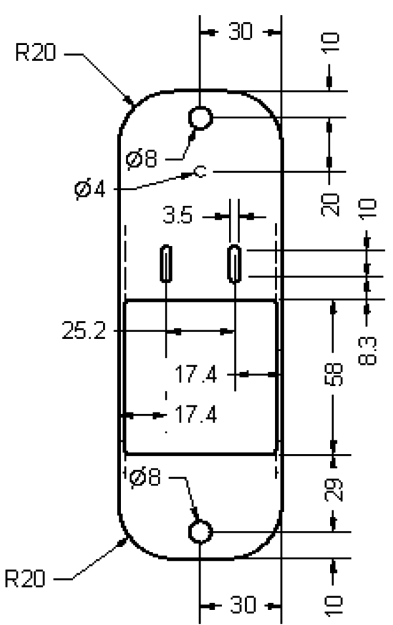

b)

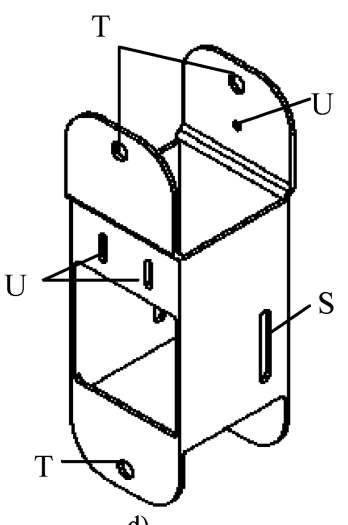

d)
Figure 4 Mechanical drawings of a standard module: (a) front view; (b) side view; (c) top view; (d) 3D view. (S: slot for fixing frame of motor; T: hole for revolute joint; $\mathrm{U}$ : hole for fixing the limit switch.)
$\mathrm{H}, \mathrm{I}, \mathrm{L}, \mathrm{N}, \mathrm{P}, \mathrm{Q}$ and $\mathrm{R}$ in Figure 3 are commercial offthe-shelf parts. The components A, B and C in Figure 3 are obtained by using a standard module that has been designed at LARM as shown in the Figure. The components $\mathrm{F}, \mathrm{M}$ and $\mathrm{O}$ in Figure 3 are obtained by a simple manufacturing process from standard aluminium bars. This assembly solution has been designed as based on previous experiences that are reported in (Ceccarelli et al., 2002; Riberioux, 2004; Carbone et al., 2005; Cigola et al., 2005; Shrot, 2006).

Six legs having the design that is shown in Figures 2-4 have been assembled on a specifically designed base frame for building the prototype of low-cost easy-operation Cassino Hexapod that is shown in Figure 5. The overall cost of this built prototype has been less than 5000 Euros, including 18 commercial low-cost DC motors, 24 limit switches, 36 pulleys, 18 belts, about 150 screws of various sizes, PLC, manufacturing of modules and other spare parts.

Aluminium alloy has been used as the material for constructing the hexapod and hence leads to savings in weight and cost of the robot. It can fit a cuboid of $60 \mathrm{~cm} \times$ $60 \mathrm{~cm} \times 50 \mathrm{~cm}$ without any payload or PLC installation. The weight of the robot without any payload or PLC installation is $17.7 \mathrm{~kg}$. The weight of the hexapod with PLC and other circuits is $21.6 \mathrm{~kg}$.

The operation of the Cassino Hexapod has been achieved by using PLC to control the different motors of the hexapod. Siemens PLC Simatic S7-200, which is fixed onboard the hexapod, is used for the operation of the

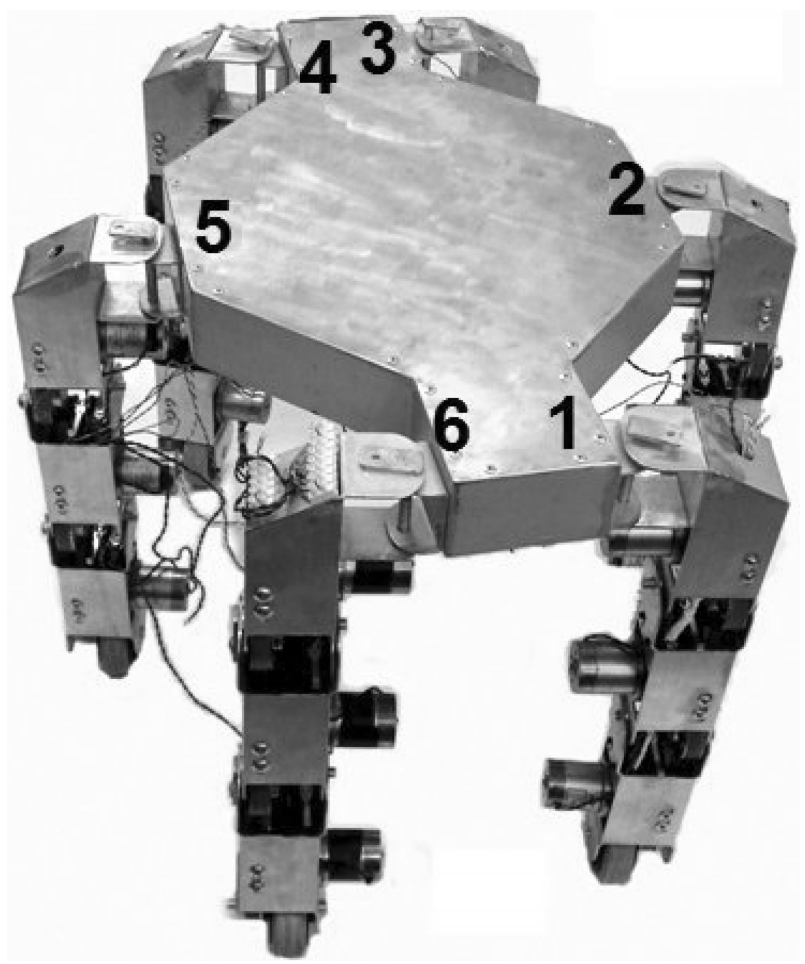

Figure 5 The assembled Cassino Hexapod prototype at LARM. 


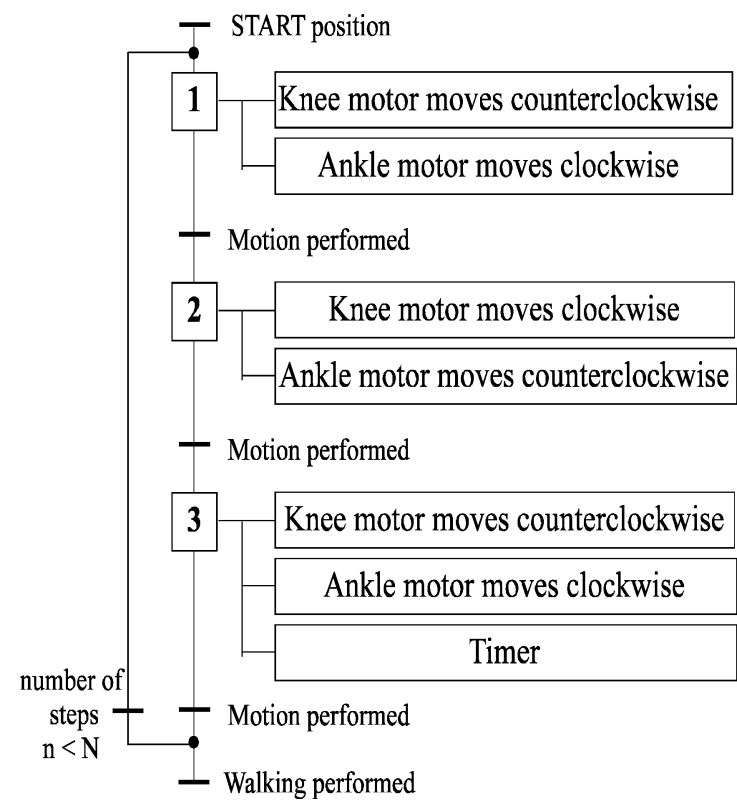

Figure 6 Descriptive Grafcet for forward and backward motion of one leg.

Cassino Hexapod. The program for the hexapod operation has been written in a windows PC which has STEP7/Micro WIN 32 installed into it. The program from the PC can be downloaded onto the flash memory of the PLC using a RS 232/PPI Cable.

\section{OPERATION STRATEGY AND PROGRAMMING}

The operation of a single leg is the most basic strategy and is operated in conjunction with other legs to perform complex walking tasks. The Grafcet logic for the movement of a single leg has been designed as shown in Figure 6 . First the knee motor is actuated in the counterclockwise direction and simultaneously the ankle motor is actuated in the clockwise direction. The motion of the motors is stopped when they reach the joint extreme positions, which is sensed by their respective limit switches ( $\mathrm{X}_{i}$ in Fig. 2a). The leg moves to its rightmost configuration, as shown in Figure 7(b).

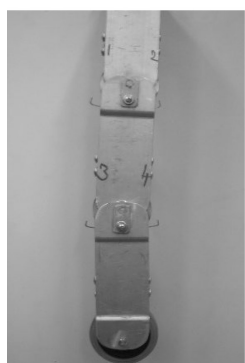

(a)

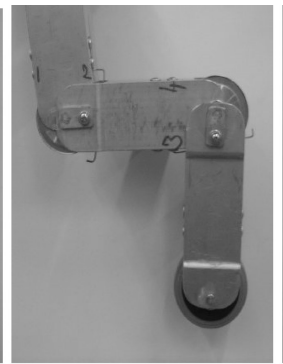

(b)

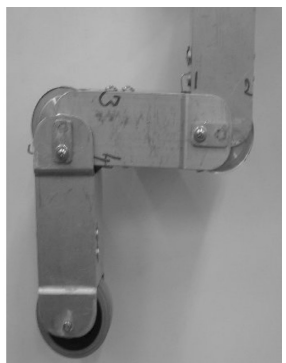

(c)
Figure 7 Robotic leg of the hexapod showing the extreme limits of its motion: (a) the leg in the straightened position; (b) the leg in the rightmost position; (c) the leg in the leftmost position.

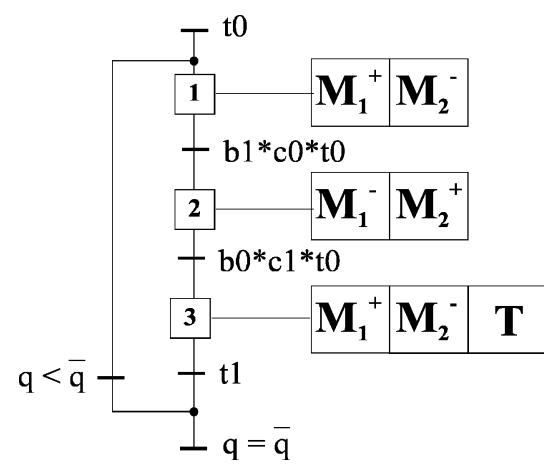

Figure 8 Functional Grafcet of forward and backward motion of a leg.

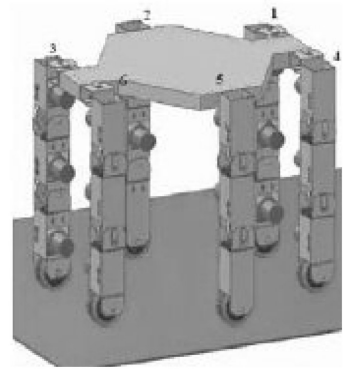

a)

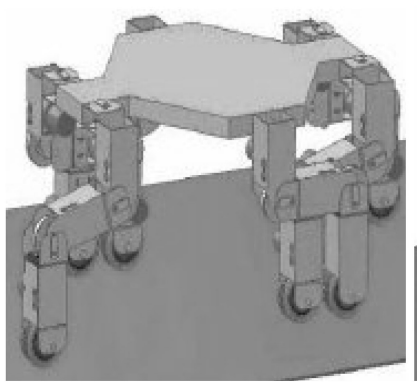

c)

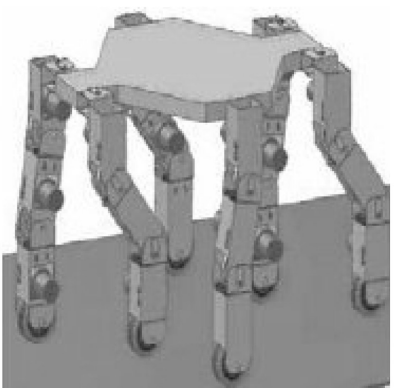

e)

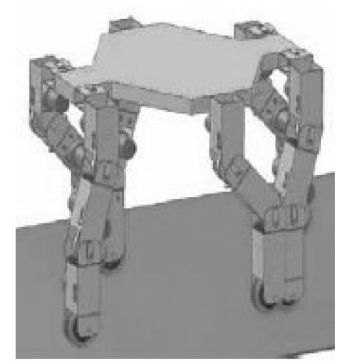

b)

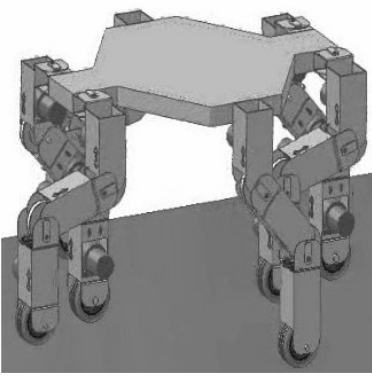

d)

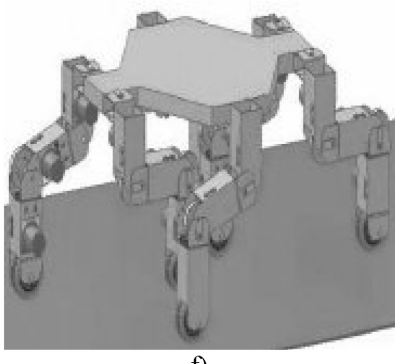

f)
Figure 9 Phases for one step double tripod forward walking motion of a hexapod robot according to the Grafcet diagram: (a) initial configuration; (b) three legs move forward; (c) three legs reach the maximum forward configuration and the other three start moving backward; (d) three legs are in the maximum forward configuration and the other three legs reach the maximum backward configuration; (e) three legs keep the maximum backward configuration and the other three move backward; (f) final configuration. 


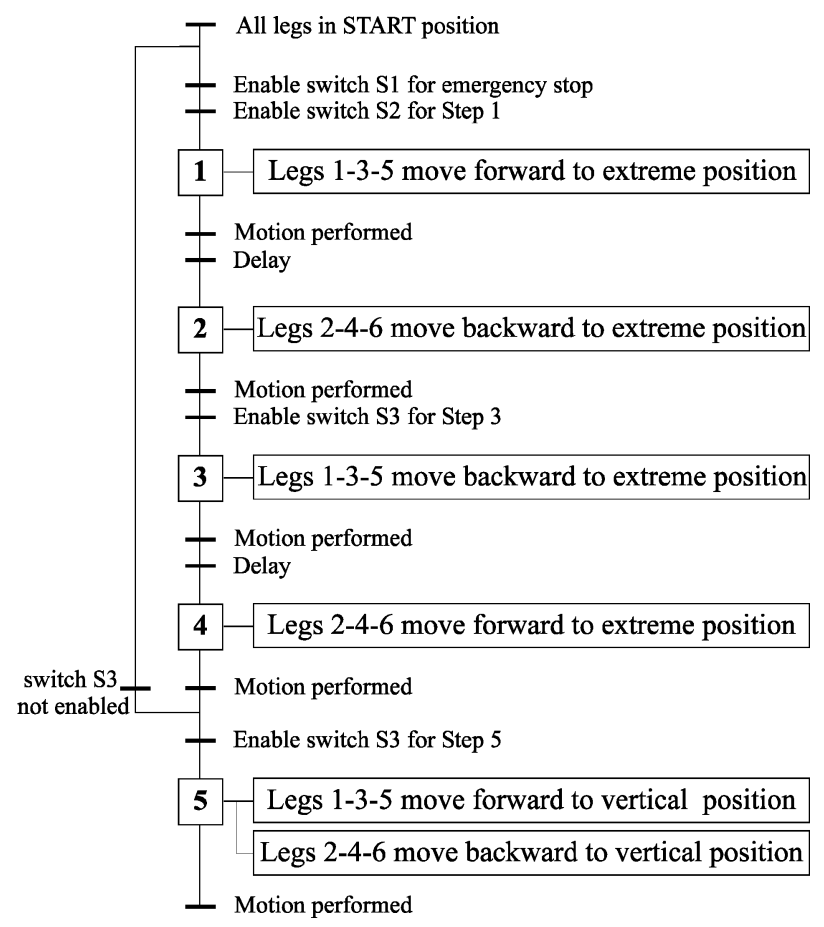

Figure 10 Descriptive Grafcet for forward walking operation of the hexapod.

Thereafter, the knee motor can be activated in the clockwise direction and ankle motor in the counterclockwise direction. The motion is stopped again at the extreme joint positions. At this point in time, the leg is in the leftmost position as shown in Figure 7(c).

In order to bring back the leg to the vertical position, the knee motor has to be moved in the counterclockwise direction and the ankle motor has to be moved in the clockwise direction. The motors are actuated for a certain period of time till the leg attains the vertical configuration. This time period is a function of voltage provided for actuation of the

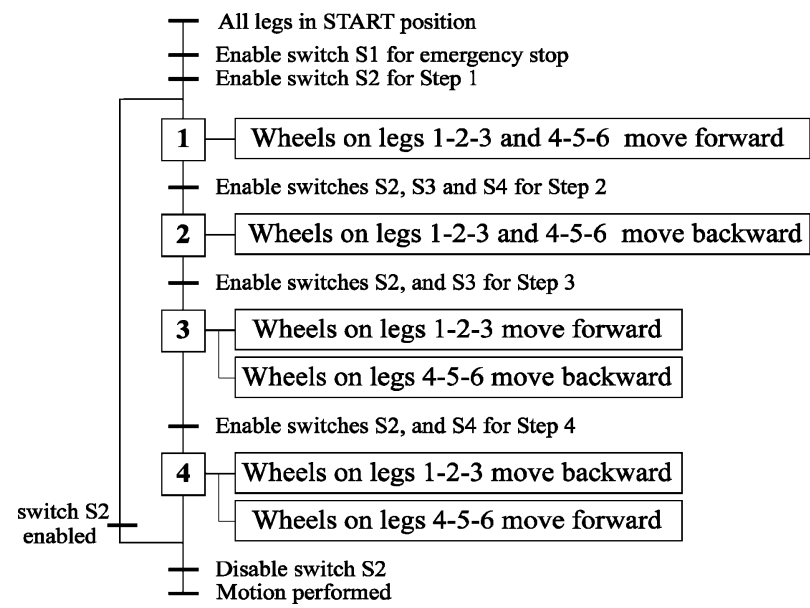

Figure 11 Descriptive Grafcet for wheel operation of the hexapod robot; forward, backward and turning operations are achieved by selecting a proper combination of latching switches $\mathrm{S} 3$ and $\mathrm{S} 4$. joint, and is determined experimentally. This scheme of operation of one leg has been shown in Figures 6 and 8 as suitable flowcharts for flexible programming. In Figure 8, $\mathrm{M}_{1}$ refers to the status of the motor $\mathrm{R}_{2}$ in the first module of the leg and it can be indicated as + for the counterclockwise motion and as - for the clockwise direction. Motor $\mathrm{M}_{2}$ refers to the status of motor $\mathrm{R}_{3}$ in the second module of the leg. A timer $\mathrm{T}$ is also provided for timing purposes. The signals $t, b$ and $c$ can be given from switches. When signal $b$ is 1 , the leg moves fully forward, when signal $c$ is 1 , the leg moves fully backward and $t$ represents the timer status. When $t$ is 0 , the timer is off and when $t$ is 1 , the timer is on. The signals $b$ and $c$ cannot be simultaneously 1 , because the leg cannot move in both the directions at once. The variable $q$ represents the internal position. A series of such elementary movements can be used for performing forward walking using tripod gait by synchronising the leg operation. In this case, the legs $1-3-5$ and 2-4-6 move simultaneously. It must be noted that, in order to ensure that the robot remains stable, at least three of the legs of the robot must be in contact with the ground at a time. Thus first, the legs $1-3-5$ are activated and are lifted in the forward direction (Fig. 9b). The legs 1-3-5 then move to forward extreme position and the legs 2-4-6 start moving in the backward direction (Fig. 9c). After this, the legs

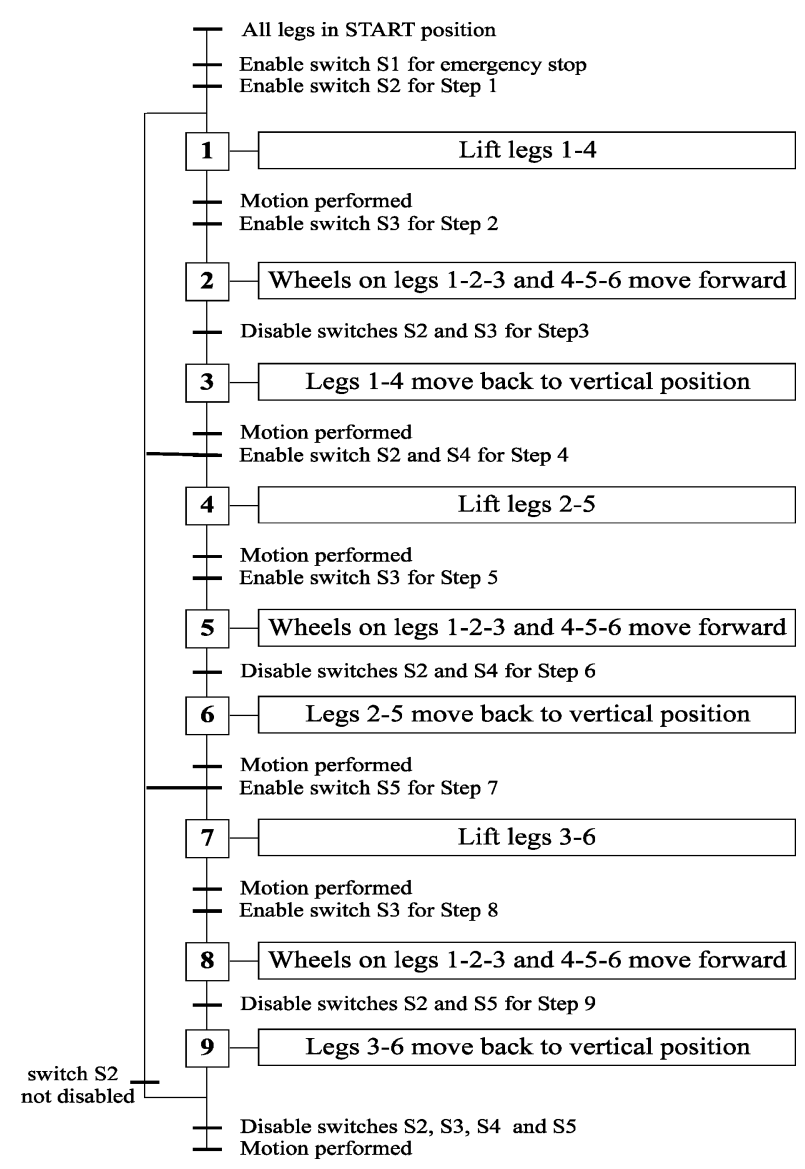

Figure 12 Descriptive Grafcet for obstacle avoidance operation. 
2-4-6 are further brought to the backward extreme position (Fig. 9d). This constitutes the start of one step of the hexapod. Now, the legs $1-3-5$ are again activated and brought backward (Fig. 9e). Then the legs 2-4-6 are brought forward (Fig. 9f). This signifies the end of the step. At this point in time, the legs are straightened back to the default position (Fig. 9a). A descriptive Grafcet for forward movement of the hexapod has been shown in Figure 10.

Wheel motion of the hexapod robot can be used for its locomotion in flat surfaces. When all the wheels of the robot move in the forward direction, the robot will move forward and when all of them move in the backward direction, the robot moves back. Differential turning of the wheels can be used to turn the robot in the right or left directions. The Grafcet logic for this operation has been presented in Figure 11 . The robot can be made to turn in the right direction, if the wheels of legs 1-2-3 are moved forward and wheels of legs $2-4-6$ are moved backward. Similarly, the robot can be turned left, by moving the wheels of legs 1-2-3 in the backward direction and the wheels of legs 2-4-6 in the forward direction.

A strategy for avoiding obstacles has also been studied. First of all, the two front legs 1-4 of the hexapod are lifted

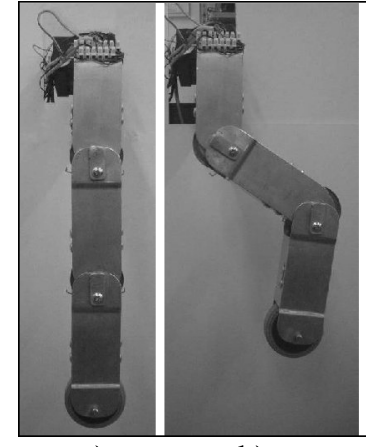

a)

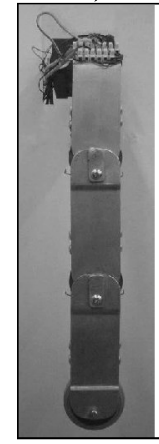

e)

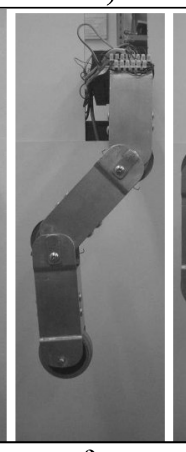

f)

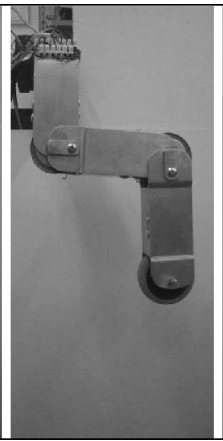

c)

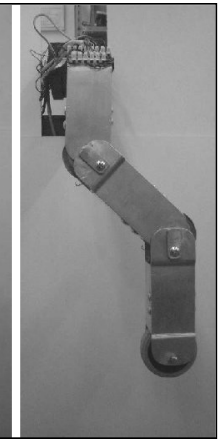

d)

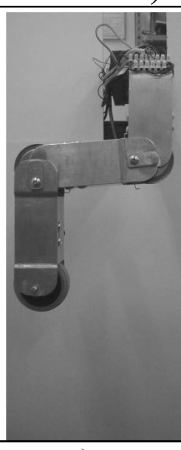

g)

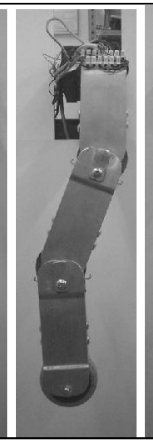

h)

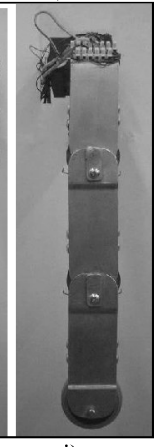

i)
Figure 13 Sequence of images showing a forward and backward motion test for one leg according to the Grafcet of Figures 8 and 9: (a) initial straight leg configuration; (b) intermediate forward configuration; (c) maximum forward configuration; (d) intermediate forward configuration; (e) intermediate straight leg configuration; (f) intermediate backward configuration; (g) maximum backward configuration; (h) intermediate backward configuration; (i) final straight leg configuration. simultaneously and the robot is moved forward using the wheels. Legs 1-4 are then brought to the ground and the middle legs 2-5 are lifted from the ground. The robot again moves forward using wheels. Finally, legs 2-5 are brought to ground and legs 3-6 are lifted. The robot moves forward again using wheels and the legs 3-6 are brought to vertical position. The Grafcet logic for obstacle avoidance has been shown in Figure 12.

\section{LABORATORY TESTS}

In order to test the working of the robotic leg, the operation of one leg of the hexapod was tested first. In this test, the leg moves from one limit position to another. Activation of switch S2, moves the leg towards left and activation of the switch S3, moves the leg towards right, according to the Grafcet in Figures 6 and 8. The images from the actual tests have been presented in Figure 13.

Then, experimental tests have been conducted on the whole prototype to verify the operation of the hexapod. For conducting the tests, the prototype has been fixed on a platform, such that none of the legs have any of interference

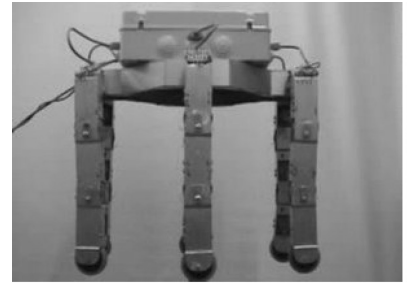

a)

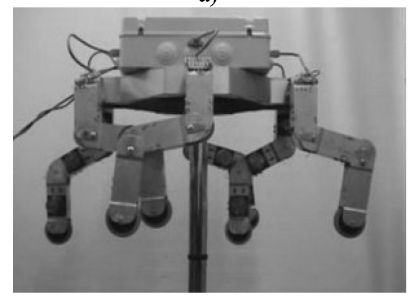

c)

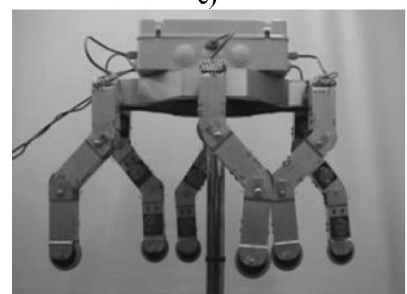

e)

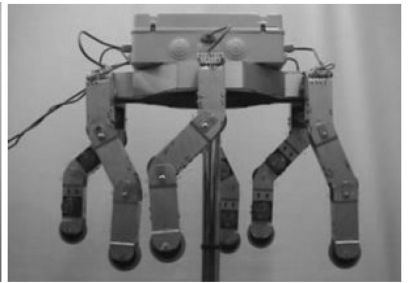

b)

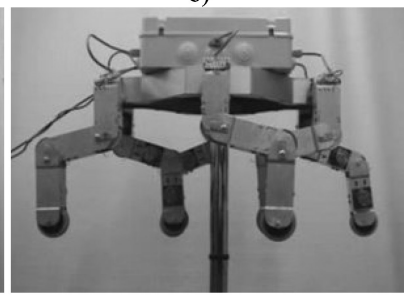

d)

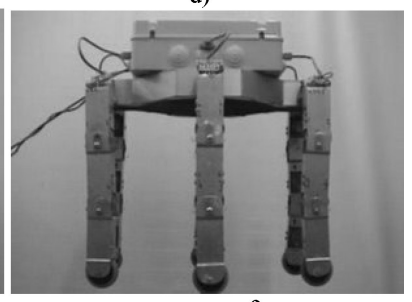

f)
Figure 14 Sequence of images showing a forward walking test of Cassino Hexapod on a fixed base: (a) initial straight leg configurations; (b) 1-3-5 legs in intermediate forward configuration and 2-4-6 legs in intermediate backward configuration; (c) 1-3-5 legs in forward configuration and 2-4-6 legs in backward configuration; (d) 2-4-6 legs in forward configuration and 1-3-5 legs in backward configuration; (e) 2-4-6 legs in intermediate forward configuration and 1-3-5 legs in intermediate backward configuration; (f) final straight leg configurations. 
from any external object. The sequential pictures from the experimental test have been presented in Figure 14.

Experimental tests have also been conducted on the prototype to test the wheeled operation of the hexapod. The hexapod has been placed on a flat surface and moved using the wheels. The hexapod has also been turned in the left and right directions, using differential motion of its wheels. The sequence of pictures from the test has been presented in Figure 15. The experimental tests that have been carried out show a suitable operation of the

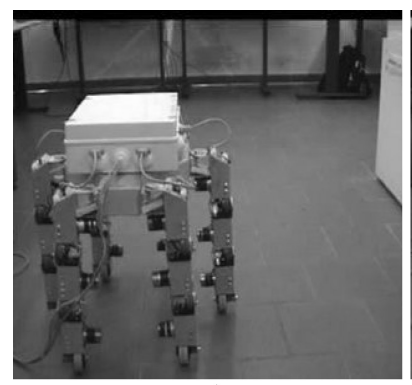

a)

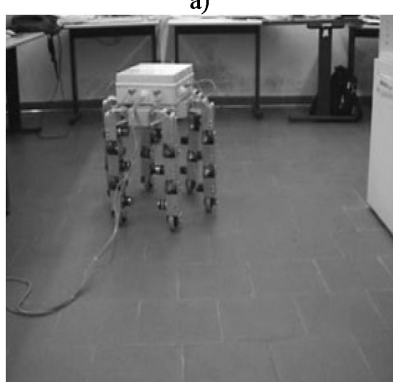

c)

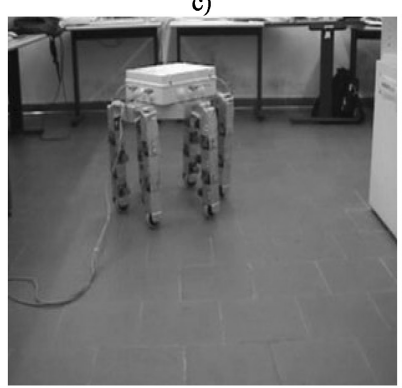

e)

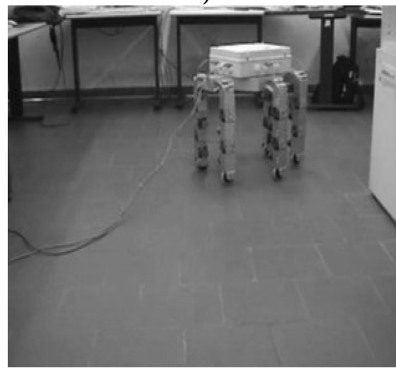

g)

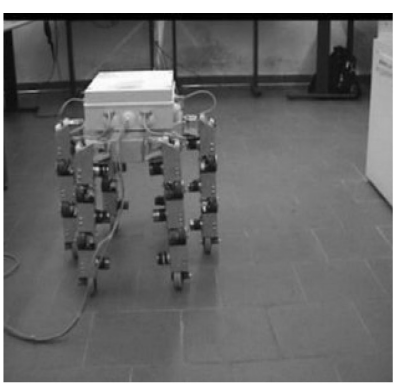

b)

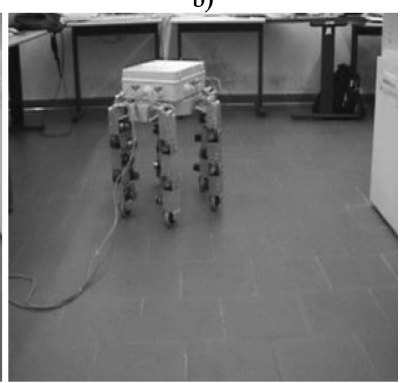

d)

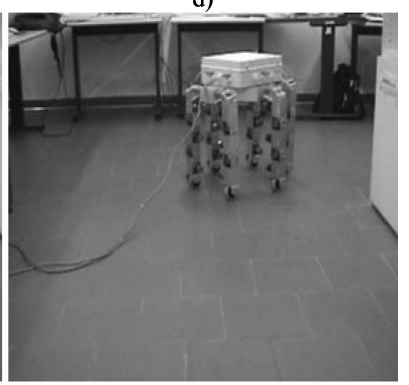

f)

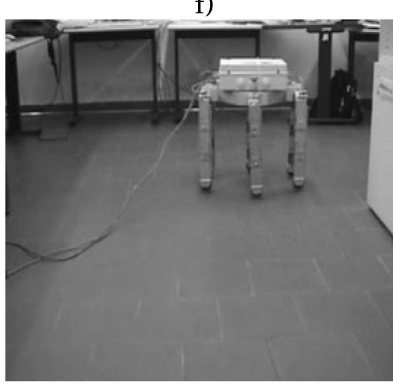

h)
Figure 15 Sequence of images showing a wheel operation test of Cassino Hexapod: (a) initial position; (b) forward motion intermediate position; (c) initial turning position $\left(15^{\circ}\right)$;

(d) second turning position $\left(30^{\circ}\right)$; (e) third turning position $\left(45^{\circ}\right)$; (f) fourth turning position with forward motion $\left(60^{\circ}\right)$; (g) fifth turning position $\left(75^{\circ}\right)$; (h) final position $\left(90^{\circ}\right)$. built prototype. However, there are certain issues such as existence of backlash in the joints, which remain an open problem. Further work will be carried out for improving the built prototype also by considering dynamic effects, as proposed for example in (Gonzalez de Santos et al., 1998).

\section{CONCLUSIONS}

The Cassino Hexapod prototype has been described by focusing on its main mechanical design and hardware characteristics that have been implemented for achieving lowcost and easy-operation features. Operation strategies for Cassino Hexapod have been proposed and implemented based on a commercial PLC unit with on-off logic. A first prototype has been built and preliminary tests have been performed with satisfactory results regarding its motion capability and easiness of motion programming and operation.

\section{ACKNOWLEDGEMENT}

The second author wishes to thank the Department of Mechanical Engineering, Indian Institute of Technology Madras, for permitting him to conduct a summer research project from April to July 2006 at LARM in Cassino.

\section{REFERENCES}

Berns K. Walking machine catalogue. Available at http://www.walking-machines.org/. (Accessed March 2007.)

Carbone G, Ceccarelli M. 2004a. Design and operation of a low-cost anthropomorphic wheeled leg for walking machines. In Proceedings of CLWAR/EURON Workshop on Robots in Entertainment, Leisure and Hobby ELH'04, Vienna, pp. 73-8.

Carbone G, Ceccarelli M. 2004b. A mechanical design of a low-cost easy-operation anthropomorphic wheeled leg for walking machines. Int 7 Robotica Manag, 9(2):3-8.

Carbone G, Ceccarelli M. 2005. Legged robotic systems. In Cutting Edge Robotics ARS Scientific Book, Wien, pp. 553-76.

Carbone G, Ceccarelli M, Molinaro F, Ottaviano E. 2005. A modular design of an easy-operation robotic leg. In Proceedings of 2nd European Conference on Mobile Robots ECMR'05, Ancona, pp. 230-5.

Ceccarelli M, Cigola M, Ottaviano E, Gallozzi A, Carbone G, Pelliccio A. 2002. A study of feasibility of using robots in architecture analysis and survey of a historical pavement. In Proceedings of 11th International Workshop on Robotics in Alpe-Andria-Danube Region RAAD 2002, Belanthofüred, pp. 113-8.

Chakraborty N, Ghosal A. 2004. Kinematics of wheeled mobile robots on uneven terrain. Mech Mach Theory, 39:12731287.

Cigola M, Pelliccio A, Salotto O, Carbone G, Ottaviano E, Ceccarelli M. 2005. Application of robots for inspection and restoration of historical sites. In Proceedings of 22nd International Symposium on Automation and Robotics in Construction ISARC 2005, Ferrara, Paper 37. 
Gonzalez de Santos P, Jimenez MA, Armada MA. 1998. Dynamic effects in statically stable walking machines. $\mathcal{F}$ Intelligent Robotic Syst, 23(1):71-85.

Hirose and Yoneda Robotic Lab Webpage. Tokyo Institute of Technology, TITAN XI. Available at http://www-robot.mes.titech.ac.jp/robot/ walking/titan11/titan11_e.html.

Hirose S, Yoneda K, Tsukagoshi H. 1997. TITAN VII: Quadruped walking and manipulating robot on a steep slope. In Proceedings of the International Conference on Robotics and Automation ICRA'97, Albuquerque, pp. 494-500.

Horodinca M, Doroftei I, Mignon E, Preumont A. 2002. A simple architecture for in-pipe inspection robots. In Proceedings of International Colloquium on Mobile and Autonomous Systems, Magdeburd, Germany.

Intelligent Machines and Special Robotics Institute Webpage. Helsinki University of Technology, WORKPARTNER. Available at http://www.automation.hut.fi/IMSRI/workpartner/.

Kawaguchi Y, Yoshida I, Iwao K, Kikuta T. 1995. Development of internal gas pipe inspection robot. $\mathcal{F}$ Robotics Mechatronics, 7(5):371-6.

LARM Webpage. Available at http://webuser.unicas.it/weblarm/ larmindex.htm.
Molinaro F. 2005. Construction and experimental validation of a robotic leg for a low cost hexapod robot. Thesis, LARM, University of Cassino, Cassino, Italy.

Rachkov M, Marques L, Almeida A. 2005. Multisensor demining robot. Autonomous Robots, 18(3):275-291.

Riberioux A. 2004. An anthropomorphic leg: Design and operation. LARM Internal Report, University of Cassino, Cassino, Italy.

Rosheim ME. 1994. Robot Evolution: The Development of Anthrobotics. A Wiley-Interscience Publication, New York.

Salmi S, Halme A. 1996. Implementing and testing a reasoning-based free gait algorithm in the six-legged walking machine Mecant. Control Eng Practice, 4(4):487-92.

Shrot A. 2006. Design and operation of Cassino low-cost hybrid hexapod robot. LARM Internal Report, University of Cassino, Cassino, Italy.

Siemens Website. Available at http://www.siemens.com.

Wilcox B, Matthies L, Genery D. 1992. Robotic vehicles for planetary exploration. In Proceedings of the 1992 International Conference on Robotics and automation, Nice, France.

Zhao YS, Lu L, Zhao TS, Du YH, Huang Z. 2000. Dynamic performance analysis of six-legged walking machines. Mech Mach Theory, 35(1):155-63.

Zielinska T, Heng J. 2002. Development of a walking machine: Mechanical design and control problems. Mechatronics, 12(5):737-54. 

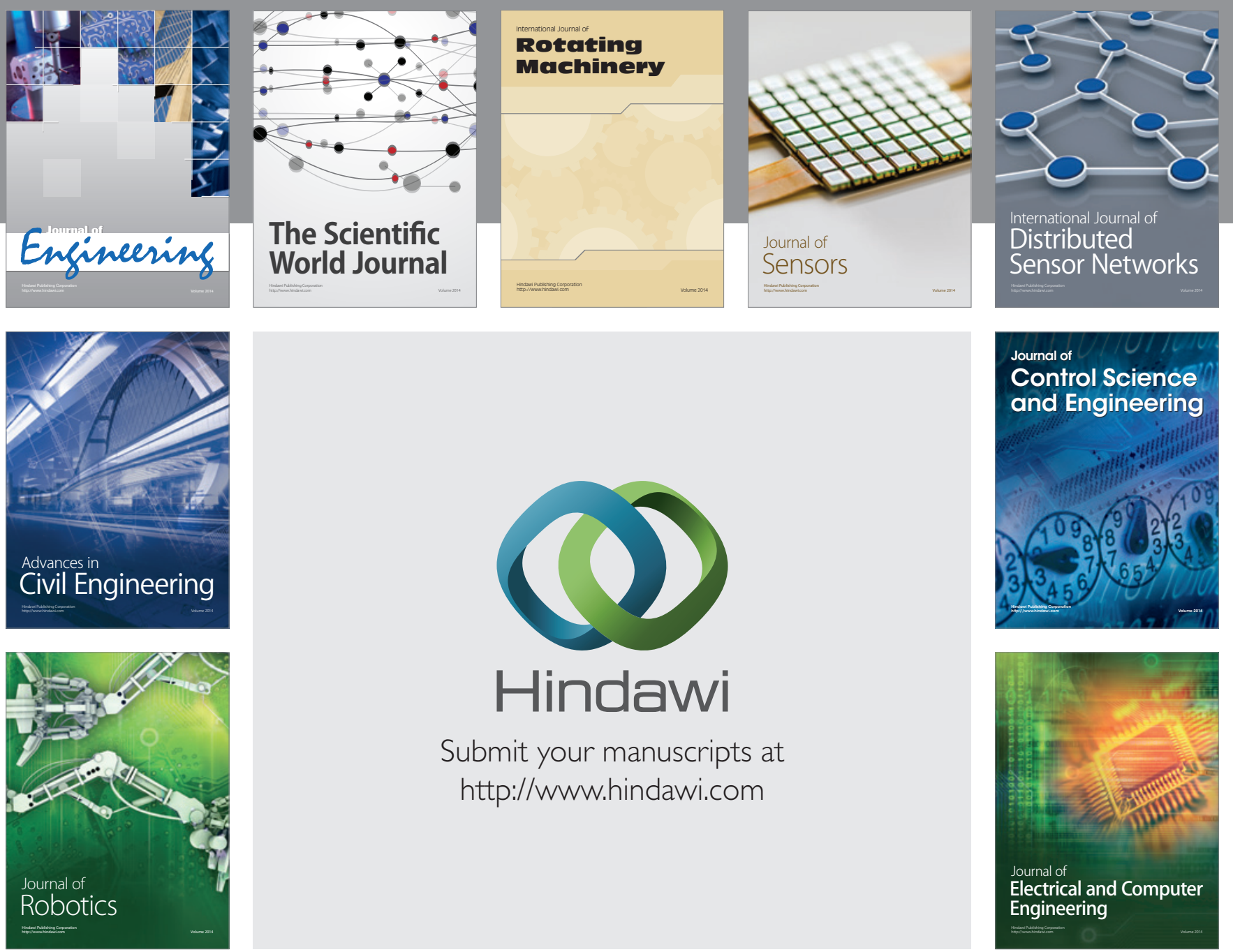

Submit your manuscripts at

http://www.hindawi.com
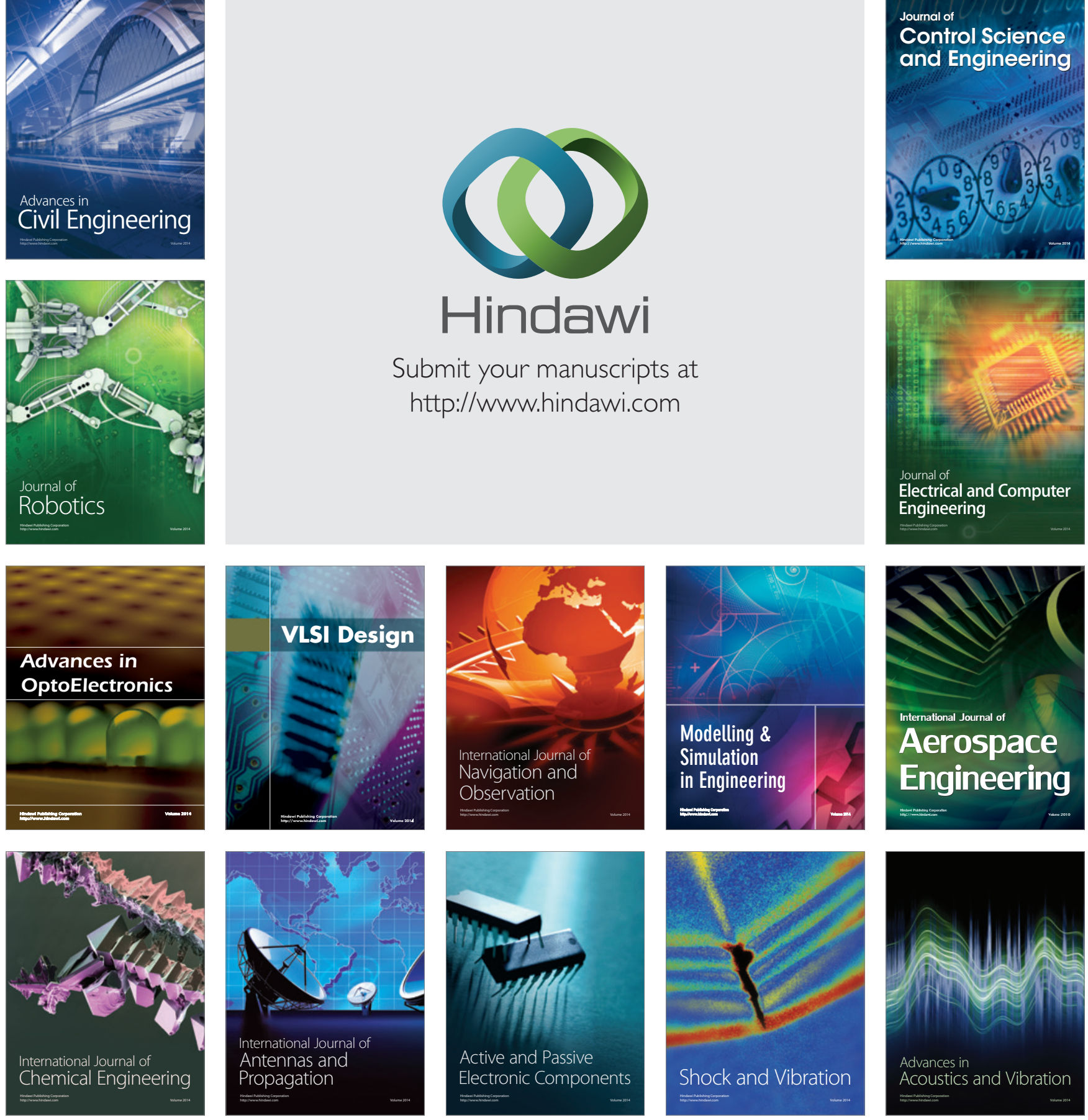